\title{
A REMARK ON THE 2-DIMENSIONAL MODULI SPACES OF VECTOR BUNDLES ON K3 SURFACES
}

\author{
TAKeshi ABE
}

\section{Introduction}

In [4], Mukai proved that a surface component of a moduli space of vector bundles on a $K 3$ suface is a $K 3$ surface if we take a general polarization. In this note, we shall consider what happens to the moduli space if we take a special polarization. Our main theorem (Theorem3.3) says that the surface component of the moduli space of semistable sheaves on a $K 3$ surface has a surjective biratonal morphism from a $K 3$ surface. We also investigate the singularity of the moduli space in some cases. The tool we shall use is the moduli of twisted semistable sheaves introduced by Matsuki and Wentworth [2]. By taking suitable $\mathbb{Q}$-divisor $L$, we obtain a natural morphism from the moduli space of $L$-twisted stable sheaves to the moduli space of semistable sheaves. Considering the curves on the moduli space of $L$-twisted stable sheaves which contract to a point on the moduli space of semistable sheaves, we can prove the main theorem.

We shall explain the content of this paper. In section 2, we fix our notations. In section 3, we state the main theorem of this paper. In section 4 , we recall Matsuki-Wentworth's twisted stability, which plays an important role in the proof of the main theorem. Section 5 is devoted to the proof of the main theorem.

\section{Notation}

$X$ always denotes a projective $K 3$ surface over $\mathbb{C}$ and $H$ is a fixed ample line bundle on $X$. As in [4], we put

$$
\tilde{H}(X, \mathbb{Z})=H^{0}(X, \mathbb{Z}) \oplus H^{2}(X, \mathbb{Z}) \oplus H^{4}(X, \mathbb{Z}) .
$$

For a coherent sheaf $F$ on $X$,

$$
\begin{aligned}
v(E) & =\operatorname{ch}(F) \sqrt{\operatorname{td}(X)} \in \tilde{H}(X, \mathbb{Z}) \\
& =\operatorname{rank}(F)+c_{1}(F)+\left\{\operatorname{rank}(F)+\frac{1}{2}\left(c_{1}(F)^{2}-2 c_{2}(F)\right)\right\} .
\end{aligned}
$$

We call $v(F)$ the Mukai vector of $F$. The symmetric bilinear form $($,$) on$ $\tilde{H}(X, \mathbb{Z})$ is defined by the following formula:

$$
(\alpha, \beta)=\alpha^{2} \cup \beta^{2}-\alpha^{0} \cup \beta^{4}-\alpha^{4} \cup \beta^{0} \in H^{4}(X, \mathbb{Z}) \simeq \mathbb{Z},
$$

Received January 18,2000. 
for $\alpha=\left(\alpha^{0}, \alpha^{2}, \alpha^{4}\right), \beta=\left(\beta^{0}, \beta^{2}, \beta^{4}\right)$. By Riemann-Roch theorem, we have $\left(v\left(F_{1}\right), v\left(F_{2}\right)\right)=\operatorname{dim} \operatorname{Ext}^{1}\left(F_{1}, F_{2}\right)-\operatorname{dim} \operatorname{Hom}\left(F_{1}, F_{2}\right)-\operatorname{dim} \operatorname{Hom}\left(F_{2}, F_{1}\right)$.

For a torsion-free sheaf $F$ on $X, F$ is said to be $H$-stable [resp. $H$-semistable] if $F$ is stable [resp. semistable] with respect to $H$ in Gieseker-Maruyama's sense. $F$ is said to be $H$-slope-stable [resp. $H$-slope-semistable] if $F$ is $\mu$-stable [resp. $\mu$-semistable] with respect to $H$.

$P_{F}(n):=\chi\left(F \otimes H^{\otimes n}\right) / \operatorname{rank} F$

$M_{H}(v):=$ the moduli space of $H$-stable sheaves with Mukai vector $v$.

$\bar{M}_{H}(v):=$ the moduli space of $H$-semistable sheaves with

$M_{H}^{\otimes L}(v):=$ the moduli space of $L$-twisted $H$-stable sheaves with Mukai vector $v$.

$\bar{M}_{H}^{\otimes L}(v):=$ the moduli space of $L$-twisted

\section{Statement of main theorem}

In this section we state the main theorem of this paper after reviewing Mukai's beautiful theorem.

On the moduli space of vector bundles on a $K 3$ surface, the following Mukai's beautiful theorem is well-known.

Theorem 3.1 (Mukai $[4])$. Let $\tilde{H}(X, \mathbb{Z}) \ni v$ be isotropic. Assume that (ด) every $H$-semistable sheaf $F$ with $v(F)=v$ is $H$-stable. Then $\bar{M}_{H}(v)$ is a K3 surface if it is not empty.

In general, without the assumption $(\boldsymbol{\uparrow}), \bar{M}_{H}(v)$ has singularities. The following gives us such an example.

Example 3.2. Let $\pi: X \rightarrow \mathbb{P}^{1}$ be an elliptic fibration with a section. Let $X \supset s$ be a section of $\pi$ and $f$ a fiber of $\pi$. Assume that $\operatorname{Pic}(X)=\mathbb{Z} s \oplus \mathbb{Z} f$. Put $H:=s+5 f$ and $v:=(2, H, 2) \in \tilde{H}(X, \mathbb{Z})$. Then $H$-semistable sheaves $E$ with $v(E)=v$ are one of the following types:

(i) $E$ fits into a nonsplit short exact sequece $0 \rightarrow \mathcal{O}(3 f) \rightarrow E \rightarrow \mathcal{I}_{x} \otimes \mathcal{O}(s+$ $2 f) \rightarrow 0$ for $x \in X \backslash s$.

(ii) $E$ is $S$-equivalent to $\mathcal{O}(4 f) \oplus \mathcal{O}(s+f)$.

Therefore $\bar{M}_{H}(v)$ is isomorphic to the surface obtained by contracting s on $X$ and has $A_{1}$-singularity.

The following is the main theorem of this paper.

Theorem 3.3. Let $v=(r, l, s) \in \tilde{H}(X, \mathbb{Z})$ be primitive and isotropic. Assume that $M_{H}(v) \neq \phi$. Then $\bar{M}_{H}(v)$ is an irreducible 2-dimensional scheme which is regular in codimension one and there exist a $K 3$ surface $S$ and a surjective birational morphism $f: S \rightarrow \bar{M}_{H}(v)$. Moreover if $x \in \bar{M}_{H}(v)$ corresponds to an $S$-equivalence class represented by a properly $H$-semistable sheaf $G_{1} \oplus \cdots \oplus G_{m}$, where $G_{1}, \cdots, G_{m}$ are $H$-stable and $P_{G_{1}}(n)=\cdots=P_{G_{m}}(n)$ and $G_{i} \neq G_{j}$ for $i \neq j$, then $\bar{M}_{H}(v)$ is normal at $x$. When $m=2, x$ is an $A_{1}$-singular point. When $m=3, x$ is an $A_{2}$-singular point. 
Remark 3.4. The author does not know whether $\bar{M}_{H}(v)$ is always normal or not.

\section{Twisted stability}

In this section we recall the definition of twisted stability (which is equivalent to the notion of $a$-stability introduced by Ellingsrud and Göttsche [1]) and wall of the ample cone defined by Matsuki-Wentworth [2] which play an important role in the proof of Theorem3.3. We fix $c_{1} \in \operatorname{Num}(\mathrm{X})$ and $c_{2} \in \mathbb{Z}$ in this section.

Definition 4.1. A hyperplane $W$ in $\operatorname{Num}(X)_{\mathbb{R}}$ is called a wall if there exist an ample line bundle $A$, an $A$-slope-semistable sheaf $E$ with $c_{1}(E)=c_{1}, c_{2}(E)=c_{2}$ and a saturated subsheaf $F$ of $E$ with $0<\operatorname{rank} F<\operatorname{rank} E$ such that

$$
\begin{gathered}
\frac{c_{1}(F)}{\operatorname{rank} F}-\frac{c_{1}(E)}{\operatorname{rank} E} \not \equiv 0 \\
\left(\frac{c_{1}(F)}{\operatorname{rank} F}-\frac{c_{1}(E)}{\operatorname{rank} E}\right) \cdot A=0 \\
W=\left\{x \in \operatorname{Num}(X)_{\mathbb{R}} \mid\left(\frac{c_{1}(F)}{\operatorname{rank} F}-\frac{c_{1}(E)}{\operatorname{rank} E}\right) \cdot x=0\right\}
\end{gathered}
$$

For a fixed ample line bundle $A$, a hyperplane $V$ in $\operatorname{Num}(X)_{\mathbb{R}}$ is called a subwall around $A$ if there exist an $A$-slope-semistable sheaf $E$ with $c_{1}(E)=c_{1}, c_{2}(E)=c_{2}$ and a saturated subsheaf $F$ of $E$ with $0<\operatorname{rank} F<\operatorname{rank} E$ such that (1) and (2) hold and that

$$
V=\left\{x \in \operatorname{Num}(X)_{\mathbb{R}} \mid\left(\frac{c_{1}(F)}{\operatorname{rank} F}-\frac{c_{1}(E)}{\operatorname{rank} E}\right) \cdot x+\frac{\chi(F)}{\operatorname{rank} F}-\frac{\chi(E)}{\operatorname{rank} E}=0\right\}
$$

Walls describe when the moduli space $\bar{M}_{H}(v)$ changes as $H$ moves in $\operatorname{Num}(X)_{\mathbb{R}}$. Subwalls tell us when the moduli space $\bar{M}_{H}^{\otimes L}(v)$ changes as $L$ moves in $\operatorname{Num}(X)_{\mathbb{R}}$, where $\bar{M}_{H}^{\otimes L}(v)$ is the moduli space of $L$-twisted $H$-semistable sheaves defined below. For details, see [2].

Definition 4.2. Let $E$ be a torsion-free sheaf on $X$ and $L$ a $\mathbb{Q}$-divisor. $E$ is said to be $L$-twisted $H$-stable [resp. $L$-twisted $H$-semistable] if for every subsheaf $F$ of $E$ with $0<\operatorname{rank} F<\operatorname{rank} E$ we have $P_{F}(n)+\left(c_{1}(F) \cdot L\right) / \operatorname{rank} F<P_{E}(n)+$ $\left(c_{1}(E) \cdot L\right) / \operatorname{rank} E \quad\left[\operatorname{resp} . P_{F}(n)+\left(c_{1}(F) \cdot L\right) / \operatorname{rank} F \leq P_{E}(n)+\left(c_{1}(E) \cdot L\right) / \operatorname{rank} E\right]$ for $n>>0$.

\section{Proof of theorem 3.3}

Lemma 5.1. Let $v=(r, l, s)$ be a primitive isotropic vector of $\tilde{H}(X, \mathbb{Z})$. Assume that there exists an $H$-stable sheaf $E$ with $v(E)=v$. If $G_{1}, \cdots, G_{m}(m \geq$ 2) are $H$-stable sheaves and $k_{1}, \cdots, k_{m}$ are positive integers such that $v=$ $\sum_{i=1}^{m} v\left(G_{i}\right)^{\oplus k_{i}}$ and $G_{i} \not G_{j}$ for $i \neq j$ and $P_{G_{1}}(n)=\cdots=P_{G_{m}}(n)=P_{E}(n)$, then we have $\left(v\left(G_{i}\right)^{2}\right)=-2$ for $1 \leq i \leq m$. Moreover the matrix $\left(\left(v\left(G_{i}\right), v\left(G_{j}\right)\right)\right)$ 
is negative semi-definite and for $\left(a_{1}, \cdots, a_{m}\right) \in \mathbb{Z}^{\oplus m}$ we have $\left(\left(a_{1} v\left(G_{1}\right)+\cdots+\right.\right.$ $\left.\left.a_{m} v\left(G_{m}\right)\right)^{2}\right)=0$ if and only if $\left(a_{1}, \cdots, a_{m}\right)$ is a multiple of $\left(k_{1}, \cdots, k_{m}\right)$.

Proof. Claim 1. $\left(v, v\left(G_{j}\right)\right)=0$ for $1 \leq j \leq m$.

Proof of Claim 1. We have $0=\left(v^{2}\right)=\sum\left(v(E), v\left(G_{j}\right)\right)$. By the stability of $E$ and the semistability of $G_{i}$, we have $\left(v(E), v\left(G_{j}\right)\right) \geq 0$. Hence the claim1 holds true.

Assume that $\left(v\left(G_{1}\right)^{2}\right) \geq 0$. We have $0 \leq\left(\left(v-\frac{r}{\operatorname{rank} G_{1}} v\left(G_{1}\right)\right)^{2}\right)=(l-$ $\left.\frac{r}{\operatorname{rank} G_{1}} c_{1}\left(G_{1}\right)\right)^{2} \leq 0$ by Hodge index. Thus $l \equiv \frac{r}{\operatorname{rank} G_{1}} c_{1}\left(G_{1}\right)$ by Hodge index theorem. We have $v=\frac{r}{\operatorname{rank} G_{1}} v\left(G_{1}\right)$. This contradicts the primitivity of $v$. Therefore we have $\left(v\left(G_{i}\right)^{2}\right)=-2$ by the stability of $G_{i}$. In the rest of this proof, we assume that $m \geq 3$. The case when $m=2$ is easy.

Claim 2. For $\left(a_{1}, \cdots, a_{m}\right) \in \mathbb{Z}^{\oplus m}$, we have $\left(\left(a_{1} v\left(G_{1}\right)+\cdots+a_{m} v\left(G_{m}\right)\right)^{2}\right) \leq 0$. If $0 \leq a_{i} \leq k_{i}$ and $\left(a_{1}, \cdots, a_{m}\right) \neq(0, \cdots, 0),\left(k_{1}, \cdots, k_{m}\right)$, then $\left(\left(a_{1} v\left(G_{1}\right)+\right.\right.$ $\left.\left.\cdots+a_{m} v\left(G_{m}\right)\right)^{2}\right)<0$.

Proof of Claim 2. We may assume that $a_{i} \geq 0$ for all $i$. $\left(v-r\left(a_{1} v\left(G_{1}\right)+\right.\right.$ $\left.\left.\cdots+a_{m} v\left(G_{m}\right)\right) /\left(a_{1} \operatorname{rank} G_{1}+\cdots+a_{m} \operatorname{rank} G_{m}\right)\right)^{2}=\left(l-r \cdot c_{1}\left(G_{1}^{\oplus a_{1}} \oplus \cdots \oplus\right.\right.$ $\left.\left.G_{m}^{\oplus a_{m}}\right) /\left(a_{1} \operatorname{rank} G_{1}+\cdots+a_{m} \operatorname{rank} G_{m}\right)\right)^{2} \leq 0$ by Hodge index theorem. The latter statement follows from the primitivity of $v$. The proof of Claim2 is completed.

By Claim 2, we have $\left(v\left(G_{i}\right), v\left(G_{j}\right)\right)=0$ or 1 for $i \neq j$. Let $\Gamma$ be a graph with vertices $v_{1}, \cdots, v_{m}$ such that $v_{i}$ and $v_{j}$ are connected by one edge if $\left(v\left(G_{i}\right), v\left(G_{j}\right)\right)=1$ and disconnected if $\left(v\left(G_{i}\right), v\left(G_{j}\right)\right)=0$. By the primitivity of $v, \Gamma$ is a connected graph, which implies the last statement.

Remark 5.2. By the characterization of Cartan matrices, we know that the ma$\operatorname{trix}\left(-\left(v\left(G_{i}\right), v\left(G_{j}\right)\right) / 2\right)$ in the above lemma is a Cartan matrix of $\tilde{A}_{n}, \tilde{D}_{n}, \tilde{E}_{6}, \tilde{E}_{7}$ or $\tilde{E}_{8}$.

Lemma 5.3. $\bar{M}_{H}(v)-M_{H}(v)$ is a finite set.

Proof. It is obvious from Lemma 5.1.

We fix a $\mathbb{Q}$-divisor $L$ near $H$ in $\operatorname{Num}(X)_{\mathbb{R}}$ so that there is no point except for $H$ on the segment connecting $L$ and $H$ which lies on a wall or a subwall around $H$.

Lemma 5.4. If $F$ is an L-twisted $H$-stable sheaf with $v(F)=v$, then $F$ is $H$-semistable. In particular, there exists a natural morphism $f: M_{H}^{\otimes L}(v) \rightarrow$ $\bar{M}_{H}(v)$.

Proof. Assume that $F$ is an $L$-twisted $H$-stable sheaf with $v(F)=v$ which is not $H$-semistable. There is a saturated subsheaf $G$ of $F$ such that:

$$
\begin{aligned}
\frac{c_{1}(G) \cdot H}{\operatorname{rank} G} & =\frac{c_{1}(F) \cdot H}{\operatorname{rank} F} \\
\frac{\chi(G)}{\operatorname{rank} G} & >\frac{\chi(F)}{\operatorname{rank} F}
\end{aligned}
$$


Since $F$ is $L$-twisted $H$-stable, we have

$$
\frac{\chi(G)}{\operatorname{rank} G}+\frac{c_{1}(G) \cdot L}{\operatorname{rank} G}<\frac{\chi(F)}{\operatorname{rank} F}+\frac{c_{1}(F) \cdot L}{\operatorname{rank} F}
$$

The two inequalities (4) and (5) contradicts the choice of $L$.

Lemma 5.5. If $F$ is an L-twisted $H$-semistable sheaf with $v(F)=v$, then $F$ is $L$-twisted $H$-stable. In particular, $\bar{M}_{H}^{\otimes L}(v)=M_{H}^{\otimes L}(v)$ is a K3 surface.

Proof. The proof of the former statement is quite similar to that of lemma5.4. We leave it to the reader. The latter follows from Corollary4.6 in [4]. (There the proof is given only for $H$-stable sheaves, but it is valid for $L$-twisted $H$-stable ones.)

Lemma 5.6. $f: M_{H}^{\otimes L}(v) \rightarrow \bar{M}_{H}(v)$ is surjective.

Proof. We employ the argument used in Proposition4.4 in [4]. Let $\mathcal{E}$ is a quasi-universal sheaf on $X \times M_{H}^{\otimes L}(v)$ with similitude $\sigma$. Given a properly $H$ semistable sheaf $G_{1} \oplus \cdots \oplus G_{m}$, where $G_{1}, \cdots, G_{m}$ are $H$-stable and $P_{G_{1}}(n)=$ $\cdots=P_{G_{m}}(n)$, we shall show that there exists an $E \in M_{H}^{\otimes L}(v)$ which is Sequivalent to $G_{1} \oplus \cdots \oplus G_{m}$. By Grothendieck-Riemann-Roch formula, $\Phi(F):=$ $\operatorname{ch}\left(\sum(-1)^{i} \operatorname{Ext}_{\pi_{M}}^{i}\left(\mathcal{E}, \pi_{X}^{*} F\right)\right)$ does not depend on the choice of $F$ with $v(F)=v$, where $\pi_{M}: X \times M_{H}^{\otimes L}(v) \rightarrow M_{H}^{\otimes L}(v)$ and $\pi_{X}: X \times M_{H}^{\otimes L}(v) \rightarrow X$. For $F \in M_{H}^{\otimes L}(v), \Phi(F)=\sigma \cdot$ (fundamental class). If $\operatorname{Hom}\left(F, G_{1} \oplus \cdots \oplus G_{m}\right)=$ $\operatorname{Hom}\left(G_{1} \oplus \cdots \oplus G_{m}, F\right)=0$ for any $F \in M_{H}^{\otimes L}(v)$, then $\Phi\left(G_{1} \oplus \cdots \oplus G_{m}\right)=0$. Therefore we have $\operatorname{Hom}\left(F, G_{i}\right) \neq 0$ or $\operatorname{Hom}\left(G_{i}, F\right) \neq 0$ for some $F \in M_{H}^{\otimes L}(v)$ and $1 \leq i \leq m$. Say we have $\operatorname{Hom}\left(G_{1}, F\right) \neq 0$ and let $\alpha$ be a nonzero element of $\operatorname{Hom}\left(G_{1}, F\right)$. Then $\alpha$ is injective and $v\left(F_{1}\right)=v\left(G_{2} \oplus \cdots \oplus G_{m}\right)$, where $F_{1}:=\operatorname{coker} \alpha$. By lemma5.1, $\left(v\left(G_{2} \oplus \cdots \oplus G_{m}\right)^{2}\right)<0$. Therefore we have $\operatorname{Hom}\left(F_{1}, G_{i}\right) \neq 0$ or $\operatorname{Hom}\left(G_{i}, F_{1}\right) \neq 0$ for some $2 \leq i \leq m$. Continuing this argument, we know that $F$ is S-equivalent to $G_{1} \oplus \cdots \oplus G_{m}$.

Lemma 5.7. If $x \in \bar{M}_{H}(v)$ corresponds to an $S$-equivalence class represented by a properly $H$-semistable sheaf $G_{1} \oplus \cdots \oplus G_{m}$, where $G_{1}, \cdots, G_{m}$ are $H$-stable and $P_{G_{1}}(n)=\cdots=P_{G_{m}}(n)$ and $G_{i} \neq G_{j}$ for $i \neq j$, then $\bar{M}_{H}(v)$ is normal at $x$.

Proof. Let $U$ be an open neighborhood of $x$. Since $U$ is constructed as a categorical quotient of a subscheme $R$ of a quot scheme, it suffices to check the normality of $R$. A point $q$ of $R$ corresponds to a surjective morphism $\mathcal{O}^{\oplus N} \rightarrow E \otimes H^{\alpha}$, where $\alpha$ is a fixed large number, $N=\operatorname{dim} H^{0}\left(X, E \otimes H^{\alpha}\right)$ and $E$ is $H$-semistable.

Using the assumption that $G_{i} \neq G_{j}$ for $i \neq j, E$ is one of the following:

(i) $E$ is simple.

(ii) $E$ is isomorphic to $F_{1} \oplus \cdots \oplus F_{l}$, where $F_{i}$ is simple for any $i$ and $l \geq 2$.

Let $Z$ be the closed subset of $R$ which consists of the points corresponding to a surjective morphism $\mathcal{O}^{\oplus N} \rightarrow E \otimes H^{\alpha}$, where $E$ is of type (ii) in the above.

Claim 3. $R \backslash Z$ is smooth, connected and of dimension $N^{2}+1$. 
Proof of Claim 3. The obstruction of smoothness at $q=\left[\mathcal{O}^{\oplus N} \rightarrow E \otimes H^{\alpha}\right]$ of $R$ (we denote it by ob $(q))$ is in $\operatorname{Ext}^{1}\left(K, E \otimes H^{\alpha}\right)$, where $K:=\operatorname{ker}\left(\mathcal{O}^{\oplus N} \rightarrow E \otimes H^{\alpha}\right)$. In [3], it is proved that $\mathrm{ob}(q)$ is mapped to 0 by the morphism $\operatorname{Ext}^{1}\left(K, E \otimes H^{\alpha}\right) \rightarrow$ $H^{2}\left(X, \mathcal{O}_{X}\right)$ which is the composite of $\operatorname{Ext}^{1}\left(K, E \otimes H^{\alpha}\right) \rightarrow \operatorname{Ext}^{2}(K, K)$ and $\operatorname{Ext}^{2}(K, K) \stackrel{t r}{\rightarrow} H^{2}\left(X, \mathcal{O}_{X}\right)$. Therefore the obstruction theory at $q$ has dimension $\operatorname{dim} \operatorname{Ext}^{1}\left(K, E \otimes H^{\alpha}\right)-1$. Since $E$ of the type (i) is simple and $\operatorname{dim} \operatorname{Ext}^{1}\left(K, E \otimes H^{\alpha}\right)-1=\operatorname{dim} \operatorname{Hom}(E, E)-1, R \backslash Z$ is smooth. Moreover for $q=\left[\mathcal{O}^{\oplus N} \rightarrow E \otimes H^{\alpha}\right]$ in $R \backslash Z, \operatorname{dim}_{q} R=\operatorname{dim} \operatorname{Hom}\left(K, E \otimes H^{\alpha}\right)=N^{2}+1$. Let $U$ be the open set of $R \backslash Z$ which consists of the points corresponding to a surjective morphism $\mathcal{O}^{\oplus N} \rightarrow E \otimes H^{\alpha}$, where $E$ is $H$-stable. Since $H$-stable sheaves are $L$-twisted $H$-stable and $M_{H}^{\otimes L}(v)$ is connected, $U$ is connected. We can easily see that $\operatorname{dim}(R \backslash(Z \cup U))<N^{2}+1$. Hence $R \backslash Z$ is connected. The proof of Claim 3 is completed.

Since $R \backslash Z$ is dense in $R, R$ is irreducible. In order to prove the normality of $R$, we use the Serre criterion. Let $q=\left[\mathcal{O}^{\oplus N} \rightarrow E \otimes H^{\alpha}\right]$ be a point in $Z$ and put $K:=\operatorname{ker}\left(\mathcal{O}^{\oplus N} \rightarrow E \otimes H^{\alpha}\right)$. We have

$$
\begin{aligned}
\operatorname{dim} \hat{\mathcal{O}}_{q, R} & \geq \operatorname{dim} \mathrm{T}_{q, R}-\left(\operatorname{dim} \operatorname{Ext}^{1}\left(K, E \otimes H^{\alpha}\right)-1\right) \\
& =-\left(v(K), v\left(E \otimes H^{\alpha}\right)\right)+1 \\
& =N^{2}+1
\end{aligned}
$$

On the otherhand $\operatorname{dim} \hat{\mathcal{O}}_{q, R}=N^{2}+1$. Therefore the above inequality is an equality, hence $\hat{\mathcal{O}}_{q, R}$ is a locally complete intersection, in particular satisfies $\left(\mathrm{S}_{2}\right)$. It is easily seen that $\operatorname{codim}(Z, R) \geq 2$.

Now we come to the proof of the main theorem.

Proof of Theorem 3.3. Most assertions were proved in the previous lemmas. Therefore we have only to show the following:

(i) $f^{-1}(x)$ is a $(-2)$-curve for $x=G_{1} \oplus G_{2} \in \bar{M}_{H}(v)$, where $G_{1}, G_{2}$ are $H$ stable and $P_{G_{1}}(n)=P_{G_{2}}(n)$.

(ii) $f^{-1}(y)$ is a union of two $(-2)$-curves which intersect each other at one point transversely for $y=G_{1} \oplus G_{2} \oplus G_{3} \in \bar{M}_{H}(v)$, where $G_{1}, G_{2}, G_{3}$ are $H$-stable and $P_{G_{1}}(n)=P_{G_{2}}(n)=P_{G_{3}}(n)$.

The proof of (i) and (ii) are quite similar and we shall show only (ii). Let $y=G_{1} \oplus G_{2} \oplus G_{3}$ be as in (iii) above. We may assume that $\left(c_{1}\left(G_{1}\right) \cdot L\right) / \operatorname{rank} G_{1}<$ $\left(c_{1}\left(G_{2}\right) \cdot L\right) / \operatorname{rank} G_{2}<\left(c_{1}\left(G_{3}\right) \cdot L\right) / \operatorname{rank} G_{3}$. Note that we have either $(l \cdot L) / r<$ $\left(c_{1}\left(G_{2}\right) \cdot L\right) / \operatorname{rank} G_{2}$ or $(l \cdot L) / r>\left(c_{1}\left(G_{2}\right) \cdot L\right) / \operatorname{rank} G_{2}$. The arguments are similar and we consider the former case.

Claim 4. $\left(v\left(G_{i}\right), v\left(G_{j}\right)\right)=1$ for $i \neq j$

Proof of Claim 4. By lemma 5.1 and Claim1 we have $\left(v\left(G_{i}\right)^{2}\right)=-2$ and $\left(v, v\left(G_{i}\right)\right)=0$. Hence we obtain $\left(v\left(G_{i}\right), v\left(G_{j}\right)\right)=1$ for $i \neq j$. 
Since $\operatorname{dim} \operatorname{Ext}^{1}\left(G_{1}, G_{2}\right)=1$ by Claim4, there exists uniquely up to $\mathbb{C}^{\times}$a nonsplit exact sequences $0 \rightarrow G_{1} \rightarrow E_{1} \rightarrow G_{2} \rightarrow 0$. Nonsplit exact sequences $0 \rightarrow E_{1} \rightarrow E_{2} \rightarrow G_{3} \rightarrow 0$ are parametrized by $\mathbb{P}\left(\operatorname{Ext}^{1}\left(G_{3}, E_{1}\right)^{\vee}\right) \cong \mathbb{P}^{1}$ up to $\mathbb{C}^{\times}$. We obtain the following commutative diagram:

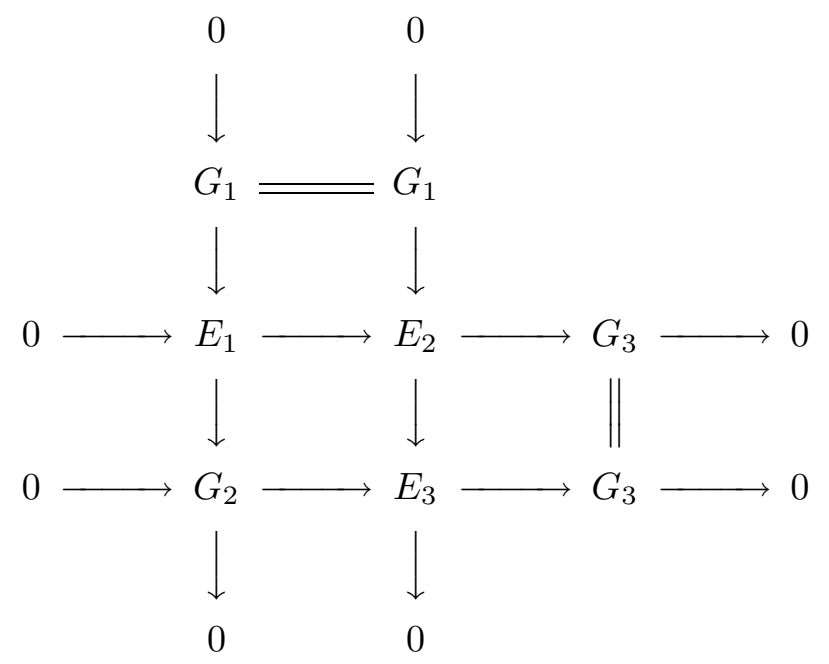

Similarly, starting from a nonsplit exact sequence $0 \rightarrow G_{1} \rightarrow E_{1}^{\prime} \rightarrow G_{3} \rightarrow 0$, we obtain the following diagram:

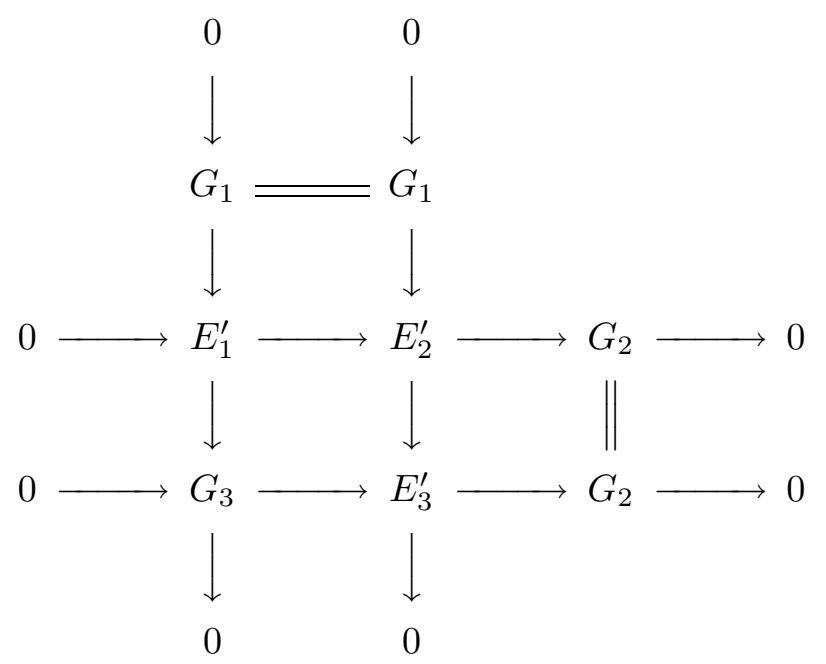

Claim 5. $E_{2}$ and $E_{2}^{\prime}$ in the above diagrams are $L$-twisted $H$-semistable. Every $L$-twisted $H$-semistable sheaf $F$ which is $S$-equivalent to $G_{1} \oplus G_{2} \oplus G_{3}$ is isomorphic to either $E_{2}$ or $E_{2}^{\prime}$ in the above.

Proof of Claim 5. The latter statement is obvious. We shall show the former statement. We check $L$-twisted $H$-semistability of $E_{2}^{\prime}$. Suppose that $E_{2}^{\prime}$ is not 
$L$-twisted $H$-semistable. We can find a saturated subsheaf $A$ of $E_{2}^{\prime}$ such that:

$$
\begin{aligned}
\frac{c_{1}(A) \cdot H}{\operatorname{rank} A} & =\frac{c_{1}\left(E_{2}^{\prime}\right) \cdot H}{\operatorname{rank} E_{2}^{\prime}} \\
\frac{c_{1}(A) \cdot L}{\operatorname{rank} A}+\frac{\chi(A)}{\operatorname{rank} A} & >\frac{c_{1}\left(E_{2}^{\prime}\right) \cdot L}{\operatorname{rank} E_{2}^{\prime}}+\frac{\chi\left(E_{2}^{\prime}\right)}{\operatorname{rank} E_{2}^{\prime}}
\end{aligned}
$$

Since $L$ is not on a subwall, we have $\chi(A) / \operatorname{rank} A=\chi\left(E_{2}^{\prime}\right) / \operatorname{rank} E_{2}^{\prime}$. This implies that $P_{A}(n)=P_{E_{2}^{\prime}}(n)$. Noting that the two exact sequences $0 \rightarrow E_{1}^{\prime} \rightarrow E_{2}^{\prime} \rightarrow$ $G_{2} \rightarrow 0$ and $0 \rightarrow G_{1} \rightarrow E_{1}^{\prime} \rightarrow G_{3} \rightarrow 0$ are nonsplit, $A$ is $S$-equivalent to $G_{1}$ or $G_{1} \oplus G_{2}$ or $G_{1} \oplus G_{3}$. In any case, we have

$$
P_{A}(n)+\frac{c_{1}(A) \cdot L}{\operatorname{rank} A}<P_{E_{2}^{\prime}}(n)+\frac{c_{1}\left(E_{2}^{\prime}\right) \cdot L}{\operatorname{rank} E_{2}^{\prime}}
$$

since we are assuming $\left(c_{1}\left(E_{2}^{\prime}\right) \cdot L\right) / \operatorname{rank} E_{2}^{\prime}<\left(c_{1}\left(G_{2}\right) \cdot L\right) / \operatorname{rank} G_{2}$. This contradicts (6) and (7). The proof of Claim 5 is completed.

By Claim 5, we obtain two morphisms $g: \mathbb{P}\left(\operatorname{Ext}^{1}\left(G_{3}, E_{1}\right)^{\vee}\right) \rightarrow M_{H}^{\otimes L}$ and $g^{\prime}: \mathbb{P}\left(\operatorname{Ext}^{1}\left(G_{2}, E_{1}^{\prime}\right)^{\vee}\right) \rightarrow M_{H}^{\otimes L}$. By construction $g$ and $g^{\prime}$ are injective. Put $C=\operatorname{Im} g$ and $C^{\prime}=\operatorname{Im} g^{\prime}$. Let $F$ be isomorphic to an $E_{2}^{\prime}$ in the above diagram. The point $[F]$ in $M_{H}^{\otimes L}(v)$ is on $C$ if and only if $\operatorname{Hom}\left(F, G_{3}\right) \neq 0$, which is equivalent to the splitness of the exact sequence $0 \rightarrow G_{3} \rightarrow E_{3}^{\prime} \rightarrow G_{2} \rightarrow 0$. It is easily seen that $0 \rightarrow G_{3} \rightarrow E_{3}^{\prime} \rightarrow G_{2} \rightarrow 0$ splits at just one point of $\mathbb{P}\left(\operatorname{Ext}^{1}\left(G_{2}, E_{1}^{\prime}\right)^{\vee}\right)$. Therefore we know $C \neq C^{\prime}$ and $C \cap C^{\prime}$ is one point. Since $C \cup C^{\prime}$ is contracted to a point of the normal surface $\bar{M}_{H}(v)$, the fact that $g$ and $g^{\prime}$ are embeddings and the transversality at $C \cap C^{\prime}$ follow from calculating the intersection matrix of $C$ and $C^{\prime}$ or more economically looking at the classification of rational double points. The proof of Theorem 3.3 is completed.

\section{Acknowledgements}

The author is grateful to Professors Masaki Maruyama, Atsushi Moriwaki and Akira Ishii for their heartful encouragement and useful advice. He also expresses thanks to Professor Hiraku Nakajima for useful comments, and the referee for pointing out several errors.

\section{References}

[1] G. Ellingsrud and L. Göttsche, Variation of moduli spaces and Donaldson invariants under change of polarization, J. Reine Angew. Math. 467 (1995), 1-49.

[2] K. Matsuki and R. Wentworth, Mumford-Thaddeus principle on the moduli spaces of vector bundles on a surface, Internat. J. Math.8 (1997), 97-148.

[3] S. Mukai, Symplectic structure of the moduli space of sheaves on an abelian or K3 surface, Invent. Math. 77 (1984), no. 1, 101-116.

[4] S. Mukai, Moduli of vector bundles on K3 surfaces I, Vector bundles on Algebraic Varieties, Oxford, 1987, 341-413. Sugaku Expositions 1 (1988), no. 2, 139-174.

Dept. of Math., Faculty of Science, Kyoto University, Kyoto, 606-8502, Japan. E-mail address: abeken@kusm.kyoto-u.ac.jp 